\title{
Anesthetic management of aortic-bicarotid double bypass graft. A case report
}

Gómez-Diago L., Reina-Giménez C., Hernández-Cádiz M.J., Alós-Zaragoza L., Ripoll-Vidal A., De Andrés J. Consorcio Hospital General Universitario de Valencia, Dept of Anaesthesiology \& Intensive Care, Valencia, Spain

Background: The perioperative management of aortic-bicarotid double bypass ( $A B D B$ ) surgery is a challenge by the limited scientific literature on that subject, the morbidity and mortality associated with the patient's condition and the complexity of the surgery and postoperative management.

Case report: 72-yrs-old male, ASA III intervened by AAA stent placement in 2010, followed up by increased size asymptomatic aortic arch aneurysm with hematoma in descending thoracic aorta. During the preoperative echo study highlights left ventricular hypertrophy with normal contractile function overall. Previous neurological exam was within normal. Surgery is planned preferential basis by the placement of an aortic prothesis bicarotid, so a partial clamping of ascending aorta and bicarotid was required.

Basic intraoperative monitoring (ECG, SpO2, EtCO2, urine output) with an arterial line placement in left radial artery before anesthetic induction to control arterial pressure and monitoring regional oxygenation saturation of brain with Invos ${ }^{\circledR}$ system (initial parameters R65/L64).

During left carotid clamping monitoring parameters sudden decresead (Invos ${ }^{\circledR}$ R70/L35) so it alerted to anesthesiasurgical team and cerebral protection measures with $100 \%$ oxygen therapy breathing and and MAP> $70 \mathrm{mmHg}$ were established obtaining improved parameters (R85/L49).

Discussion: Performing an $A B D B$ is a high-risk procedure in patients with important morbidity and mortality. Complications during surgery are frequent and early treatment should be instituted. Postoperative management requires close monitoring of the patient.

\begin{abstract}
After clamping resolution an improvement in monitoring parameters near to initial values was found.The patient's clinical stability encouraged for early extubation in order to neurological examination (slight paresis in the right arm).

At 4 hours, he had a sudden cardiac arrest, which recovered after cardiac massage and administration of adrenaline ev (1 $\mathrm{mg})$.

Major bleeding is caused through drainage tubes requiring surgical revision.A hematoma on the back part of the ascending aorta and the dissection aortic intima causing false light were evidenced this time.

The gravity of the situation and the serious complication led to the patient to die.
\end{abstract}

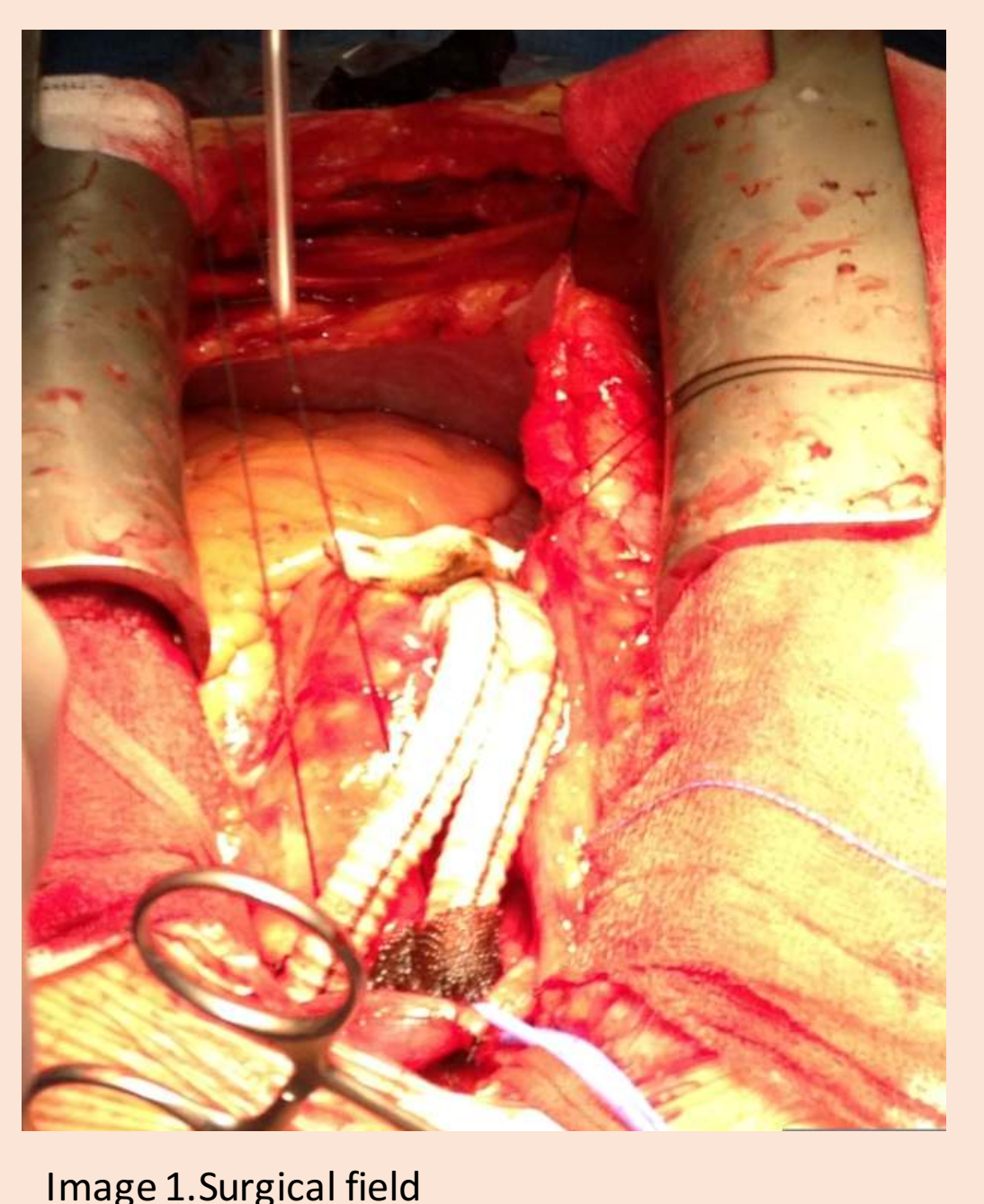

Learning points: Needing regional monitoring oxygen saturation and advanced invasive monitoring. Treating early complications. Waking up early for neurological assessment. Strict control of blood pressure and adequate analgesia. 